\title{
THE DISCRETENESS OF THE SPECTRUM OF SELF- ADJOINT, EVEN ORDER, ONE-TERM, DIFFERENTIAL OPERATORS
}

\author{
ROGER T. LEWIS
}

ABstract. An open question which was asked by I. M. Glazman is answered. It is well known that the condition

$$
\lim _{x \rightarrow \infty} x^{2 n-1} \int_{x}^{\infty} r^{-1}=0
$$

is sufficient for the discreteness and boundedness from below of the spectrum of selfadjoint extensions of $(-1)^{n}\left(r y^{(n)}\right)^{(n)}$. This paper shows that the condition is also necessary.

\section{Let $\tilde{L}$ denote any selfadjoint extension of}

$$
(-1)^{n}\left(r y^{(n)}\right)^{(n)} \quad(x \geqq 0, r(x)>0) .
$$

THEOREM 1. A necessary and sufficient condition that the spectrum of $\tilde{L}$ be discrete and bounded below is that

$$
\lim _{x \rightarrow \infty} x^{2 n-1} \int_{x}^{\infty} r^{-1}=0
$$

The proof of the sufficiency part of the above theorem is due to V. A. Tkachenko and is exhibited in a book by I. M. Glazman [2, pp. 120, 121]. Glazman states that if $r(x)$ is monotonic, then relation (1) is necessary for the discreteness of the spectrum, but in the general case the problem of the necessity of condition (1) for $n>1$ remains open. The development below completes the proof of Theorem 1 .

Define

$$
L_{2 n} y=\sum_{k=0}^{n}(-1)^{n-k}\left(p_{k} y^{(n-k)}\right)^{(n-k)}
$$

where we assume that $p_{k}$ is $n-k$ times continuously differentiable. The domain of $L_{2 n}$ is the set of all $y$ such that $y$ and $L_{2 n} y$ are elements of $L^{2}[a, b]$ for $a, b$ satisfying $0<a<b<\infty$ and $y^{(k)}$ is absolutely continuous on compact subintervals of $(0, \infty)$ for $k=0,1, \cdots, 2 n-1$. Let $\mathscr{D}$ denote

Presented to the Society, November 16, 1973; received by the editors April 9, 1973 and, in revised form, June 12, 1973.

AMS (MOS) subject classifications (1970). Primary 34B25.

(c) American Mathematical Society 1974 
the set of all $y$ in the domain of $L_{2 n}$ such that $y$ has compact support interior to $[a, \infty)$ for some $a>0$. Let $T$ denote the closure of the restriction of $L_{2 n}$ to $(2)$.

The differentiable operator $L_{2 n}$ is said to be oscillatory on $[a, \infty)$ if for any given $b>a$ there are numbers $c$ and $d$ and a function $y \neq 0$ such that $L_{2 n}(y)=0, b \leqq c<d$, and

$$
y^{(k)}(c)=0=y^{(k)}(d) \text { for } k=0,1, \cdots, n-1 .
$$

Otherwise, $L_{2 n}$ is said to be nonoscillatory on $[a, \infty)$.

THEOREM 2 (GlazmAN [2]). The following statements are equivalent.

(i) The spectrum of every selfadjoint extension of $T$ is bounded below and discrete.

(ii) For every real number $\lambda, L_{2 n}-\lambda$ is nonoscillatory on $[0, \infty)$.

TheOREM 3 (AhLBRANDT [1]). Suppose that $r$ and $p$ are positive, realvalued functions which are Lebesgue integrable on arbitrary compact subintervals of $[0, \infty)$. Then $(-1)^{n}\left(r^{-1} y^{(n)}\right)^{(n)}-p y$ is nonoscillatory on $[a, \infty)$ for some $a>0$ if and only if $(-1)^{n}\left(p^{-1} y^{(n)}\right)^{(n)}-r y$ is nonoscillatory on $[a, \infty)$.

The following theorem is an extension by the author [3] of a theorem due to Glazman [2].

THEOREM 4. If $p(x) \leqq 0,0<r(x) \leqq M x^{\alpha}$ for some $\alpha<2 n-1$, and

where

$$
\limsup _{x \rightarrow \infty} x^{2 n-1-\alpha} \int_{x}^{\infty}|p|>M \cdot A_{n}^{2}
$$

$$
A_{n}^{-1}=\frac{(2 n-1)^{1 / 2}}{(n-1) !} \sum_{k=1}^{n}(-1)^{k-1}\left(\begin{array}{l}
n-1 \\
k-1
\end{array}\right) /(2 n-k)
$$

then $(-1)^{n}\left(r y^{(n)}\right)^{(n)}+$ py is oscillatory on $[a, \infty)$ for $a>0$.

Proof of Theorem 1. (Necessity) Suppose that

then

$$
\lim _{x \rightarrow \infty} x^{2 n-1} \int_{x}^{\infty} r^{-1} \neq 0
$$

$$
\limsup _{x \rightarrow \infty} x^{2 n-1} \int_{x}^{\infty} r^{-1}=\beta>0 .
$$

There is a constant $c>0$ such that $\lambda \geqq c$ implies that $\beta>A_{n}^{2} / \lambda$. Hence, by Theorem $4,{ }^{1}(-1)^{n}\left(\lambda^{-1} y^{(n)}\right)^{(n)}-r^{-1} y$ is oscillatory on $[a, \infty)$ for $a>0$ and

\footnotetext{
1 Theorem 11/31 (9)/, p. 100 of Glazman [2] could also be used if one lets $q=-\lambda r^{-1}$.
} 
$\lambda \geqq c$ which implies that $(-1)^{n}\left(r y^{(n)}\right)^{(n)}-\lambda y$ is oscillatory on $[a, \infty)$ by Theorem 3. Consequently, by Theorem 2 , the spectrum of $\tilde{L}$ is not necessarily discrete and bounded below.

\section{REFERENCES}

1. Calvin D. Ahlbrandt, Equivalent boundary value problems for self-adjoint differential systems, J. Differential Equations 9 (1971), 420-435. MR 44 \#1860.

2. I. M. Glazman, Direct methods of qualitative spectral analysis of singular differential operators, Fizmatgiz, Moscow, 1963; English transl., Israel Program for Scientific Translations, Jerusalem, 1965 and Davey, New York, 1966. MR 32 \#2938; \#8210.

3. Roger $\mathrm{T}$. Lewis, Oscillation and nonoscillation criteria for some self-adjoint, even order, linear differential operators, Pacific J. Math. (to appear).

Department of Mathematics, Slippery Rock State College, Slippery Rock, Pennsylvania 16057 